\title{
Prevalence of cerumen impaction and associated factors among primary school children in Mwanza City, Tanzania
}

\author{
Shija B. Mabenda ${ }^{1,2}$, Gustave Bunabe ${ }^{2}$ Japhet M. Gilyoma ${ }^{2}$, Phillipo L. Chalya ${ }^{{ }^{*}}$, William Mahalu ${ }^{3}$ \\ 'Department of Surgery, Catholic University of Health and Allied Sciences, Bugando, Mwanza, Tanzania \\ ${ }^{2}$ Department of Otorhinolaryngology, Catholic University of Health and Allied Sciences, Bugando, Mwanza, Tanzania \\ ${ }^{3}$ Department of Cardiothoracic, Catholic University of Health and Allied Sciences, Bugando, Mwanza, Tanzania
}

\begin{abstract}
Background: Cerumen impaction is a worldwide problem constituting a significant proportion of health problems in many settings and its prevalence varies. There is a paucity of published data regarding this condition in Tanzania with none from Mwanza region. The aim of this study was to determine the prevalence of cerumen impaction and associated factors among primary school children in Mwanza City and to assess the effect of cerumen impaction and its removal on hearing ability

Methods and Patients: This was a cross-sectional, community based study of primary school children with cerumen impaction that was carried out in randomly selected primary schools in Mwanza City between December 2016 and May 2017. Multistage cluster sampling technique was employed to obtain a required number of the study population.

Results: Out of the 406 participants, ninety-five (23.4\%) had cerumen impacted in their ears. Of these, 56 (58.9\%) were males and $39(41.1 \%)$ were females. The mean age at presentation was $11.24 \pm 8.86$ years. Ear bud abuse (83.7\%) was the most common predisposing factor for cerumen impaction. Cerumen impaction was found in the right ear of 9 (9.5\%) patients and in the left ear in 31 (32.6\%) patients and bilateral in $55(57.9 \%)$ of patients. The major presenting symptoms were ear itching, otalgia, hearing loss and tinnitus. Ear syringing was used to remove cerumen impaction and caused significant improvement in hearing thresholds. There were no recorded complications.

Conclusion: Cerumen impaction is a common otologic presentation in our sub-region. Ignorance with the profound abuse of cotton buds is the major predisposing factor. Health education is of the essence as treatment is simple and effective.
\end{abstract}

Keywords: Cerumen impaction; prevalence; associated factors; Tanzania

\section{Introduction}

Cerumen impaction is a worldwide public health problem constituting a significant proportion of health problems in many settings and its prevalence varies (Jacob et al., 1997; Kirfi et al., 2014). Globally, the prevalence of cerumen impaction varies considerably from $8.9 \%$ to $20.0 \%$, and it is a major cause of primary care consultation and common comorbidity in otorhinolaryngological patients (Grossan, 1998; McCarter et al., 2007; Adegbiji et al., 2014). Many studies from Europe put the prevalence of cerumen impaction between $2 \%$ to $6 \%$, whereas a few studies from African continent estimated the prevalence to be from $10 \%$ to $20 \%$ (Holocomb, 2009). The prevalence also varies between different age groups. The prevalence of cerumen impaction varies greatly among population with different characteristics; approximately $10 \%$ in children, $5 \%$ in normal healthy adults, up to $57 \%$ in older patients in nursing homes and $36 \%$ within patients with mental retardation (Al-Khabori et al., 2007). In developing world like Tanzania, cerumen impaction has been reported to be the commonest ear disease or etiology of hearing impairment, with prevalence range from $8.4 \%$ to $52.6 \%$ (Minja and Machemba, 1996; Olusanya et al, 2004; Subha \& Raman, 2006; Al-Khabori et al., 2007). At Bugando

“Correspondence: E-mail plchalya65@gmail.com 
Medical Centre, cerumen impaction is one of the most common causes of consultation to the Otorhinolaryngological surgeons and its removal is the most common procedure performed at the Otorhinolaryngology clinic (Mushi et al., 2016; Bugando Medical Centre-ENT database, 2016-2017, Unpublished).

The habit of self-cleaning ears by pointed objects, ear canal abnormalities, excess sebum production, foreign body in the ear, associated ear infection and ear trauma are some of the predisposing factors for cerumen impaction (Crandell \& Roeser, 1993; Al-Khabori et al., 2007). Other factors include use of ear hearing aids, cotton swab use and use of ear protection (Crandell \& Roeser, 1993). In addition, different people appear to be at a higher risk of suffering from accumulation of cerumen, with an increased risk for the elderly, men, people with intellectual impairment and secondary-care populations (Crandell \& Roeser, 1993; Meador, 1995).

Cerumen impaction is one of the leading causes of reversible hearing loss (Olusanya et al., 2000; McCarter et al., 2007; Adoga et al., 2010; Eziyi et al., 2011). Other problems associated with cerumen impaction are pain, tinnitus, itching and dizziness but cerumen impaction with conductive hearing impairment remains the most common indication for ear syringing (Olusanya et al., 2000; McCarter et al., 2007).

Various methods used in the removal of cerumen include the use of ceruminolytic agents, curette method (using either a probe or cerumen hook) and lavage (Olusanya et al., 2000), but ear syringing is the most employed and efficient method for removing ear wax (Ogunleye \& Awobem, 2004). Complications can occur during ear syringing and these include otalgia, tympanic membrane perforations, external auditory canal lacerations, dizziness and failure to syringe wax/foreign bodies or debris out (Ogunleye \& Awobem, 2004).

Studies from the developing world have documented impacted cerumen as the commonest cause of hearing impairment among primary school children (Minja and Machemba, 1996; Olusanya et al., 2000; Olusanya et al, 2004; Eziyi et al., 2011). Previous studies have revealed an association between hearing loss and poor school performance (Olusanya et al., 2000; Olusanya et al, 2004; Eziyi et al., 2011). Despite the well documented fact that cerumen impaction is one of the commonest otolaryngology diseases that is associated with hearing impairment among primary school children and the magnitude of the problem is not known in this part of the world; there is a paucity of literature on this ailment from our environment. The study aimed to determine the prevalence of cerumen impaction and associated factors among primary school children in randomly selected primary schools in Mwanza City. The secondary objective related to this study was to assess the effect of cerumen impaction and its removal on hearing ability.

\section{Methods and Patients}

\section{Study area and design}

This was a cross-sectional, community-based study of primary school children with cerumen impaction that was carried out in randomly selected primary schools in Mwanza City between December 2016 and May 2017. Mwanza City consists of two districts namely Nyamagana and Ilemela. The City has a total of 107 primary schools comprising of 80 public and 27 private schools. Each school has standard I to VII pupils.

Study population, selection criteria and sampling procedure

The study population included primary school children from randomly selected primary schools in Mwanza City during the period of the study. The study participants included all children (from standard 
I to VII) who were diagnosed to have cerumen impaction during otoscopic examination and whose parents/caretaker consented for the study. Children with pre-existing ear problem like chronic suppurative otitis media, ear foreign body, external otitis media and those who were absent on the day of study were excluded from the study. Multistage cluster sampling technique was employed to obtain a required number of the study population (students). A sampling frame from the list of schools was prepared and used for drawing sample. From sampling frame (schools), study units (students) were sampled through simple random method until the required sample size was obtained.

\section{Study variables}

Independent variables in this study included socio-demographic characteristics (e.g. age, sex, area of residence, socio-economic status), predisposing factors, clinical presentation (such as the presenting complaint, duration of symptoms, laterality and history of any predisposing factors), associated comorbidities and cerumen impaction removal methods. Dependent variables were cerumen impaction and hearing ability.

\section{Recruitment of participants}

The recruitment of participants was conducted in randomly selected primary schools in Mwanza City. On the day of the study a quiet classroom was selected at the school premises where the examination was conducted and the ambient noise measured using a sound level meter. The examination procedure was explained and demonstrated to the children. The examination commenced with otoscopic examination of both the right and left outer and middle ear and torch light examination of dentition. Each ear was examined by pneumatic otoscopy. All abnormal findings were confirmed by a trained otolaryngologist. The diagnosis of cerumen impaction was made if there was presence of visually occluding cerumen in the external auditory canal preventing view of the tympanic membrane with or without otalgia and complaint of decrease in hearing acuity/hearing impairment. Otoscopic finding was noted and those who were found to have ceruminous impaction, audiometry was done before (control group) and after (intervention group) the remove of the impacted cerumen to determine the presence of hearing loss and results were noted. History of ear bud usage and presence of pathology like missing tooth, caries tooth and malocclusion were noted.

\section{Data collection and Statistical data analysis}

Data was collected using a pre-tested coded questionnaire. The statistical analysis was performed using statistical package for social sciences (SPSS) version 20.0 for Windows (SPSS, Chicago IL, USA). The mean (+standard deviation) and ranges were calculated for continuous variables whereas proportions and frequency tables were used to summarize categorical variables. A Chi-squared $\left(\chi_{2}\right)$ test was used to assess significance of association between the independent and dependent (outcome) variables in the categorical variables. The level of significance was considered as $p<0.05$.

\section{Results}

\section{Socio-demographic characteristics}

During the period of study, a total of 406 participants were enrolled into the study. Among these, 343 (84.5\%) were from Nyamagana district and the remaining 63 (15.5\%) participants were from llemela district in Mwanza City. Out of the 406 participants examined, ninety-five (23.4\%) had cerumen impacted in their ears. Of these, 56 (58.9\%) were males and 39 (41.1\%) were females with a male to 
female ratio of 1.4:1. Their ages ranged from 5 to 16 years with a mean of $11.24 \pm 8.86$ years. The peak age incidence was $11-15$ years accounting for $68.4 \%$ (Table 1 ).

Table 1: Age-sex distribution of children with cerumen impaction

\begin{tabular}{llll}
\hline \multirow{2}{*}{ Age group (years) } & \multicolumn{3}{c}{ Sex } \\
\cline { 2 - 3 } & Male, N (\%) & Female, N (\%) & \\
\hline $0-5$ & $0(0.0)$ & $1(1.1)$ & $1(1.1)$ \\
$6-10$ & $17(17.9)$ & $9(9.4)$ & $26(27.3)$ \\
$11-15$ & $37(38.9)$ & $28(29.5)$ & $65(68.4)$ \\
$16-20$ & $2(2.1)$ & $1(1.1)$ & $3(3.2)$ \\
\hline Total & $56(58.9)$ & $39(41.1)$ & $95(100.0)$ \\
\hline
\end{tabular}

\section{Predisposing factors among patients with cerumen impaction}

The most common predisposing factor identified was ear bud usage in 340 (83.7\%) participants. No predisposing factor was identified in $76(18.7 \%)$ patients. Ear bud usage was significantly associated with cerumen impaction $(p=0.001)$. There was no significantly association between age, gender, dental pathology and the presence of cerumen impaction $(p>0.05)$ (Table 2$)$.

Table 2: Association between demographic factors, predisposing factors and the presence of impacted cerumen

\begin{tabular}{|c|c|c|c|c|}
\hline \multirow{2}{*}{ Factor } & \multicolumn{2}{|c|}{ Cerumen impaction } & \multirow{2}{*}{ Chi-squared (X2) } & \multirow{2}{*}{ p-value } \\
\hline & Presence & Absence & & \\
\hline \multicolumn{5}{|l|}{ Age (years) } \\
\hline $6-10$ & $151(37.2 \%)$ & $27(6.6 \%)$ & 0.947 & 0.214 \\
\hline $11-15$ & $160(39.4 \%)$ & $379(93.3 \%)$ & & \\
\hline$>15$ & $2(0.5 \%)$ & $1(0.2 \%)$ & & \\
\hline \multicolumn{5}{|l|}{ Sex } \\
\hline Male & $56(13.8 \%)$ & $126(31.0 \%)$ & 0.782 & 0.423 \\
\hline Female & $39(9.6 \%)$ & $185(45.6 \%)$ & & \\
\hline \multicolumn{5}{|l|}{ Ear bud usage } \\
\hline Yes & $82(20.2 \%)$ & $258(63.5 \%)$ & 32.876 & 0.001 \\
\hline No & $13(3.2 \%)$ & $53(13.1 \%)$ & & \\
\hline \multicolumn{5}{|l|}{ Dental pathology } \\
\hline Yes & $19(4.7 \%)$ & $69(17.0)$ & 0.635 & 0.564 \\
\hline No & $76(18.7 \%)$ & $242(59.6 \%)$ & & \\
\hline
\end{tabular}

Clinical presentation among primary school pupils with cerumen impaction 
Cerumen impaction was found in the right ear of 9 (9.5\%) of patients and in the left ear in 31 (32.6\%) patients and bilateral in 55 (57.9\%) of patients. Table 3 shows sex distribution according to laterality. The majority of participants in this study were asymptomatic accounting for $50.7 \%$ of cases. The major presenting symptoms were ear itching and otalgia in $18.5 \%$ and $9.1 \%$ of patients, respectively. Table 4 shows distribution of study population according to clinical presentation. The duration of illness ranged from 1 day to 36 days with a mean of $10.02 \pm 11.71$ days.

Table 3: Sex distribution according to laterality

\begin{tabular}{lccc}
\hline \multirow{2}{*}{ Laterality } & \multicolumn{2}{c}{ Sex } & \multirow{2}{*}{ Total } \\
\cline { 2 - 3 } & Male N (\%) & Female N (\%) & \\
\hline Right ear & $7(7.4)$ & $2(2.1)$ & $9(9.5)$ \\
Left ear & $22(23.1)$ & $9(9.5)$ & $31(32.6)$ \\
Bilateral & $27(28.4)$ & $28(29.5)$ & $55(57.9)$ \\
\hline Total & $56(58.9)$ & $39(41.1)$ & $95(100.0)$ \\
\hline
\end{tabular}

Table 4: Distribution of study population according to clinical presentation

\begin{tabular}{lcc}
\hline Clinical presentation & Frequency & Percent \\
\hline Tinnitus & 22 & 5.4 \\
Hearing Loss & 35 & 8.6 \\
Ear Itching & 75 & 18.5 \\
Pain & 37 & 9.1 \\
Others & 31 & 7.6 \\
Asymptomatic & 206 & 50.7 \\
\hline Total & 406 & 100 \\
\hline
\end{tabular}

Treatment and interventions among primary school pupils with cerumen impaction

Participants found with cerumen impaction were subjected to Audiometric examination to assess their hearing thresholds. The hearing thresholds before intervention (ear syringing) ranged from 5 to $58 \mathrm{~dB}$ with a mean of $30.54 \pm 12.43 \mathrm{~dB}$ (Control group). The mean hearing thresholds after intervention (ear syringing) was $10.12 \pm 7.22 \mathrm{~dB}$ in more than two thirds of participants. The difference in mean improvement in hearing between intervention and control groups was $20.42 \mathrm{~dB}(95 \% \mathrm{Cl}=6.8$ to 26.82$)$ $(p<0.001)$. After intervention there was clinical relief of symptoms such as ear etching, hearing loss and pain. There were no documented complications after syringing

\section{Discussion}


Globally, cerumen impaction is one of the most common ear pathology treated in the otorhinolaryngological clinic (McCarter et al., 2007 ;Roland et al., 2008). There is a marked variation in the prevalence of cerumen impaction worldwide, with western countries having a low rate compared to Africa (Crandell \& Roeser, 1993). In this study, the prevalence of cerumen impaction among primary school pupils in our setting accounted for $23.4 \%$. This concurs with figures of $10 \%$ to $25 \%$ that have been reported from various parts of Africa (Man et al., 1985; Elango et al., 1991; Hatcher et al., 1995; Minja and Machemba, 1996). Our figure is higher than that of 2-6\% reported in many studies in developed countries (Crandell \& Roeser, 1993). However, a high prevalence of $46.7 \%$ was reported in Nigerian primary school pupils (Eziyi et al , 2011). Olusanya et al (2004), Adhikari et al (2008) and Adhikari et al (2009) found cerumen impaction to be a common ear disease amongst school children in Nigeria, Nepal, and Kathmandu valley with a comparable high prevalence of $52.6 \%, 62 \%$ and $60.6 \%$, respectively. Our study can be supported by the findings of Minja and Machemba (1996) and other studies (Man et al., 1985; Elango et al., 1991; Hatcher et al., 1995) with the prevalence rate varying between $8.6 \%$ and $28.2 \%$ in children of higher age group. These differences in the rate of cerumen impaction reflect differences in the prevalence and risk factors for developing cerumen impaction among different study settings.

More than two third of the study participants were in the age group of 11-15 years. This observation is in keeping with other studies which reported similar finding (Ologe et al., 2005; McCarter et al., 2007), but at variant with Kirfi et al (2014) who found cerumen impaction was more prevalent among patients under nine years of age. We could not establish the reasons for the high rate of impacted cerumen in this age group. In our study, we found no association between the incidence of cerumen impaction and age. Other studies have reported a higher incidence of cerumen impaction in the elderly, in school-aged children and in patients with mental retardation (Olusanya et al., 2000; Ologe et al., 2005; Eziyi et al., 2011).

In this study, male participants were more affected than females. The male preponderance in this study has been reported elsewhere (Kirfi et al., 2014; Gabriel, 2015). We could not find in literature the reasons for this gender differences although it has been speculated that cerumen impaction is more common in males as the tragus or hair in the external auditory canal being larger and coarser that impair the natural dislodgement of cerumen.

The association between cerumen impaction in the ear canal and the use of cotton ear bud in paediatric age group was reported to occur mainly in the left ear (Sim, 1988). Mackenin et al (1994) concluded that the use of ear bud might be associated with cerumen impaction. Cotton ear bud abuse was the most common predisposing factors identified in this study. Accumulation of excess cerumen may result from misguided attempts to remove cerumen with objects and also from the breakdown of the natural epithelial migration of the external auditory canal (Sim, 1988; Olusanya, 2003). Health education to discourage use of cotton buds alongside other objects in the ear is highly needed. The practice pushes the ear cerumen beyond the skin lined external auditory canal which subsequently gets retained and cannot be removed by the natural outward migration of the epithelium of the external auditory canal (Sim, 1988; Meyers, 1997; Olusanya, 2003). The danger of ear drum perforation, trauma to canal skin, infecting the external auditory canal amongst other health problems also remain a possibility if these wrong practices are not stopped.

Failure of the secreted cerumen to be expelled from the outer ear causes cerumen accumulation (Hatcher et al., 1995). Ear cerumen is normally expelled by chewing movements of the jaw. The movement of the lower jaw aids in expelling the cerumen. So any dental pathology that interferes with chewing movements of the jaw causes cerumen accumulation (Fransman, 2006). Dental caries is common in children. In primary school children milk teeth falls and later there is eruption of permanent teeth. So these children will not be able to chew well (Adegbiji et al., 2014). Fransman (2006) has reported that there is a strong positive relationship between cerumen impaction 
and absence of last molar tooth from his study. Therefore, children should be encouraged to chew well while eating foods.

In agreement with other studies done elsewhere (Adoga et al., 2009; Eziyi et al., 2011), our study found that bilateral ear impaction was commoner than either right or left ear affectation. Adegbiji et al (2014) documented that unilateral cerumen impaction (75.1\%) was more common than bilateral cerumen impaction. As reported in other studies (Adoga et al., 2009; Eziyi et al., 2011), more than half of participants in our study were asymptomatic and only few reported ear itching, hearing impairment, pain and tinnitus. The consequences of cerumen impaction includes complications such as otalgia, hearing impairment, tinnitus, vertigo, otitis externa apart from preventing the needed examination of the external auditory meatus and the tympanic membrane while audiologically, it affects audiometric test results or prevent testing. Studies from the developing world have documented impacted cerumen as the commonest ear disease or etiology of hearing impairment, with prevalence rates of $8.4 \%$ to 52.6\% (Olusanya et al, 2004; Subha \& Raman, 2006; Al-Khabori et al., 2007). The impacted cerumen has also been shown to cause noticeable hearing problems in school children and this is a common finding in health surveys (Subha \& Raman, 2006; Al-Khabori et al., 2007; Eziyi et al., 2011). Sharma et al (1992) and Jacob et al (1997) studies reported cerumen as the most common cause of hearing impairment, which accounted for $50.0 \%$ and $29.8 \%$ of cases respectively. Olusanya et al (2000) have reported impacted cerumen as the most common disorder in school children which has a significant association with hearing loss and school performance. Ear syringing is a common otorhinolaryngology procedure by which the external auditory canal is irrigated with saline at body temperature. Among various other options, ear syringing remains the first line procedure in management of cerumen impaction because it is safe, non-traumatic and effective (Ogunleye \& Sogebi, 2005; Adoga et al., 2009; Eziyi et al., 2011). In this study, ear syringing was used to remove cerumen impaction and caused significant improvement in hearing thresholds.

In conclusion, this study demonstrated that cerumen impaction is a public health problem amongst primary school children in Mwanza City. The high prevalence of cerumen impaction in this region with its attendant problem of hearing impairment which can lead to poor school performance is a significant health problem. Ear bud usage was significantly associated with cerumen impaction. Ear syringing was the most common mode of treatment of our patients and caused significant improvement in hearing impairment. We therefore recommend continuing medical education and proper otological examination for children at school entrance and at regular interval for the early detection. Health education to improve the low level of awareness among parents and school authorities on the consequences of wax impaction should also be embarked upon.

\section{Ethical consideration}

Ethical approval to conduct the study was obtained from the CUHAS/BMC joint institutional ethic review committee before the commencement of the study. The permission to conduct the study was sought from Regional Administrative Secretary of Mwanza City as well as Nyamagana and Ilemela District Executive Directors. Permission was also obtained from respective primary school authority. Study participants were informed on the study purpose as well as rationale and that, participation was voluntary, hence parents/caretakers willing for their children to participate in the study signed in the consent form.

Conflict of interests: The authors declare no conflict of interests

\section{References}


Adegbiji, W.A., Alabi, B.S., Olajuyin, O.A., Nwawolo, C. (2014) Earwax impaction: Symptoms, predisposing factors and perception among Nigerians. Journal of family Medicine and Primary Care 3, 379

Adoga, A.A., Bakari,A.A., Kodiya, A.M., Ahmad, B.M. (2009) Cerumen auris: Asurvey of its management at the national ear care center, Kaduna, Nigeria. Internet Journal of Otorhinolaryngology 12, 1-5.

Adhikari, P. (2009) Pattern of ear diseases in rural school children: Experiences of free health camps in Nepal. International Journal of Pediatric Otorhinolaryngology 73, 1278-80.

Al-Khabori, M., Kumar, S., Khandekar, R. (2007) Magnitude of impacted earwax in Oman, its impact on hearing impairment and economic burden of earwax on health services. Indian Journal of Medical Sciences 61, 278.

Crandell, C.C., Roeser, R.J. (1993) Incidence of excessive/impacted cerumen in individuals with mental retardation: A longitudinal investigation. American Journal of Mental Retardation 97, 568-74.

Elango, S., Purohit, G.N., Hashim, M., Hilmi, R. (1991) Hearing loss and ear disorders in Malaysian school children. International Journal of Pediatric Otorhinolaryngology 22, 75-80.

Eziyi, J., Amusa, Y., Nwawolo, C., Ezeanolue, B. (2011) Wax Impaction in Nigerian School Children. East and Central African Journal of Surgery 16, 40-5

Fransman, D. (2006) Can removal of back teeth contribute to chronic earwax obstruction?. British Journal of Learning Disabilities 34, 36-41.

Gabriel, O.T. (2015) Cerumen impaction: Challenges and management profile in anrural health facility. Niger Medical Journal 56, 390-393.

Grossan, M. (1998) Cerumen removal--current challenges. Ear, Nose \& Throat Journal 77, 541.

Hatcher, J., Smith, A., Mackenzie, I., Thompson, S., Bal, I., Macharia, I., et al. (1995) A prevalence study of ear problems in school children in Kiambu district, Kenya. International Journal of Pediatric Otorhinolaryngology 33, 197-205.

Holocomb, S.S. (2009) Get an earful of the new cerumen impaction guidelines. Journal for Nurse Practitioners 34 , 14-9.

Jacob, A., Rupa, V., Job, A., Joseph, A. (1997) Hearing impairment and otitis media in a rural primary school in South India. International Journal of Paediatric Otorhinolaryngology 39, 133-8.

Kirfi, A.M., Mainasara, G.M., Sa'idu, A.T., Fufore, M.B., Joseph, Y. (2014) Cerumen auris in Abubakar Tafawa Balewa University teaching hospital Bauchi, North-eastern Nigeria. Sudan Medical Monitor 9, 75.

Macknin, M.L., Talo, H., VanderBrug Medendorp, S. (1994) Effect of cotton-tipped swab use on earwax occlusion. Clinical pediatrics 33, 14-8.

Mann, S.B., Bhardwaj, A., Gudi, S.P., Mehra, Y.N. (1985) Incidence of speech, hearing and ENT problems in schoolgoing children. Hearing Aid Journal 2, 39-42.

McCarter, D.F., Courtney, A.U., Pollart, S.M. (2007) Cerumen impaction. American Family Physician 75, 10.

Meador, J.A. (1995) Cerumen impaction in the elderly. Journal of Gerontology Nursing 21, 43-5.

Meyers, A. O. (1997) Managing cerumen impaction. Postgraduate Medicine 62, 207-9.

Minja, B., Machemba, A. (1996) Prevalence of otitis media, hearing impairment and cerumen impaction among school children in rural and urban Dar es Salaam, Tanzania. International Journal of Paediatric Otorhinolaryngology 37, 29-34 
Mushi,M.F., Mwalutende, A.E., Gilyoma, J.M., Chalya, P.L., Seni, J., Mirambo, M.M., Mshana, S.E.(2016) Predictors of disease complications and treatment outcome among patients with chronic suppurative otitis media attending a tertiary hospital, Mwanza Tanzania. BMC Ear Nose and Throat Disorders 16, 1

Ogunleye, A.O., Sogebi, R.O. (2005) Otic foreign bodies in children in Ibadan, Nigeria. Nigeria Journal of Surgical Research 7, 305-8.

Ogunleye, A.O., Awobem, A.A. (2004) Trends of ear syringing at Ibadan, Nigeria. African Journal of Medicine and Medical Sciences 33, 35-37

Ologe, F.E., Segun-Busari, S., Abdulraheem, I.S., Afolabi, A.O. (2005) Ear diseases in elderly hospital patients in Nigeria. The Journals of Gerontology Series A Biological Sciences and Medical Sciences 60, 404-6.

Olusanya, B.O., Okolo, A., Adeosun, A. (2004) Predictors of hearing loss in school entrants in a developing country. Journal of Postgraduate Medicine 50, 173.

Olusanya, B.O. (2003) Hearing impairment in children with impacted cerumen. Annals of Tropical Paediatrics 23, 121-8.

Olusanya, B.O., Okolo, A.A., ljaduola, G.T. (2000) The hearing profile of Nigerian school children. International Journal of Pediatric Otorhinolaryngology 55, 173-9

Roland, P.S., Smith, T.L., Schwartz, S.R., Rosenfeld, R.M., Ballachanda, B., Earll, J.M., et al. (2008) Clinical practice guideline: cerumen impaction. Otolaryngology--Head and Neck Surgery 139, S1-S21.

Sharma, H., Bhusan, V., Dayal, D., Mishra, S.C. (1992) Preliminary study of hearing handicap in school-going children. Indian Journal of Otolaryngology and Head Neck Surgery 30, 119-24

Sim, D.W. (1988) Wax plugs and cotton buds. Journal of Laryngology and Otology 102, 575-6.

Subha, S.T., Raman, R. (2006) Role of impacted cerumen in hearing loss. Ear, Nose \& Throat Journal 85, 650.

Ulaganathan, M., Shalini, R. (2015) A descriptive study of prevalence of impacted wax and its predisposing factors in school children. International Journal of Healthcare and Biomedical Research 4, 136-143 